\title{
Incidence of cesarean section according to Robson's classification in the Department of Gynecology and Obstetrics of the General Hospital Dr. Fernando Quiroz Gutiérrez, ISSSTE
}

\author{
Incidencia de la operación cesárea según la clasificación de Robson en el Servicio de \\ Ginecología y Obstetricia del Hospital Gral. Dr. Fernando Quiroz Gutiérrez del Instituto de \\ Seguridad Social al Servicio de los Trabajadores del Estado
}

Aidé I. Manny-Zitle and José M. Tovar-Rodríguez*

Department of Gynecology and Obstetrics, Hospital Gral. Dr. Fernando Quiroz Gutiérrez, Instituto de Seguridad Social al Servicio de los Trabajadores del Estado (ISSSTE), Mexico City, Mexico

\begin{abstract}
Introduction: The increase of cesarean sections worldwide has increased significantly, our country is no exception, in some places, it exceeds $60 \%$ of cesarean sections in relation to vaginal delivery, there is no adequate classification for the analysis of this increase. The classification of the 10 groups of Robson is based on four pillars: a) obstetric history; b) progress of labor c) category of pregnancy; and d) gestational age. We suggest using Robson's classification to determine which group of pregnant women contribute most to the total number of cesareans in our institution. Method: This retrospective, descriptive cross-sectional, observational study was conducted from 2014 to 2016, all pregnant women with more than 27 weeks' gestation were included. Pregnancy was resolved in 706 women with a caesarean section of $65.29 \%$, mean age was $31 \pm 4.2$ years, and gestational age $38.5 \pm 6.7$ weeks. $46.74 \%$ were primiparous. The most frequent maternal indication for surgery was by iterative cesarean section and the fetal one was due to presentation dystocia, as for the location of the patients within the Robson classification was group 5 with $21.24 \%$, group 2 with $13.88 \%$ and the 1 with $9.6 \%$. Conclusion: The previous uterine scarring was the determining factor in most of the cesarean sections, we suggest to influence the indication of the first cesarean section and thus to avoid uterine scars.
\end{abstract}

KEY WORDS: Pregnancy. Caesarean section. Robson classification.

\section{Resumen}

Introducción: El número de cesáreas en todo el mundo se ha incrementado de forma importante y nuestro país no es la excepción. En algunos lugares se supera el $60 \%$ de cesáreas en relación al parto vaginal. No existe una clasificación adecuada para el análisis de este incremento. La clasificación de los 10 grupos de Robson se basa en cuatro pilares: a) antecedents obstétricos; b) progreso del parto; c) categoría del embarazo; y d) edad gestacional. Sugerimos utilizar la clasificación de Robson para determinar qué grupo de mujeres embarazadas contribuyen más al total de cesáreas en nuestra institución. Método: Estudio retrospectivo, descriptivo, transversal, observacional, de 2014 al 2016, incluyendo a todas las embarazadas con más de 27 semanas de gestación. Se resolvió el embarazo a 706 mujeres con un porcentaje de cesárea del 65.29\%. El promedio de edad fue de $31 \pm 4.2$ años, y el de la edad gestacional fue de $38.5 \pm 6.7$ semanas. Eran primigestas el $47 \%$. La indicación materna más frecuente de cirugía fue por cesárea iterativa, y la fetal por distocia de presentación. En cuanto a la ubicación del mayor

\author{
Correspondence: \\ *José M. Tovar-Rodríguez \\ Felipe Ángeles y Canario \\ Col. Bellavista, Del. Álvaro Obregón \\ C.P. 01140, Ciudad de México, México \\ E-mail: gacetajuarez@gmail.com
}

Date of reception: 13-12-2017

Date of acceptance: 24-04-2018

DOI: 10.24875/CIRUE.M18000040

Cir Cir. 2018;86:232-239

Contents available at PubMed www.cirugiaycirujanos.com 
número de pacientes dentro de la clasificación de Robson, fue el grupo 5 con un $21.24 \%$, seguido del grupo 2 con un $13.88 \%$ y del grupo 1 con un 9.6\%. Conclusión: La existencia de cicatriz uterina previa fue el factor determinante en la mayor parte de las cesáreas. Sugerimos incidir sobre la indicación de la primera cesárea y así disminuir cicatrices uterinas.

PALABRAS CLAVE: Embarazo. Cesárea. Clasificación de Robson.

\section{Introduction}

The rates of cesarean section (CS) have substantially increased, particularly in developed countries and especially over the last decade. This continuous increase has provoked a debate on which the appropriate rates of CS should be, on the right to choose or not a cesarean delivery in the absence of medical indication, on the increase of potential risks for the mother and the fetus that could result from implementing a policy for the reduction in the number of CS and on concerns about over-medicalization and excessive use of resources. The World Health Organization (WHO) declared in 1985: "There is no justification for any region to have a percentage of cesarean sections higher than $10-15 \% "$. However, general monitoring of CS rates is insufficient to understand the essence of the problem and its causes. The analysis of more in-depth information, such as which group of women or obstetric populations are undergoing CS, will shed more light on interventions that might reduce the rates of CS when and where appropriate ${ }^{2}$. The absence of internationally accepted standardized methodologies has significant hindered comparisons and monitoring of CS rates throughout the world ${ }^{3}$. In 2001, there was a proposal for action-oriented monitoring and auditing of the rate of CS: the ten-group classification proposed by Robson, which is internationally applicable and allows comparisons over time within healthcare facilities and between them. This classification provides the basis for changing practices in specific and prospectively identifiable groups of women, with a useful and clinically relevant classification system to improve the results being obtained. In addition, the ten-group classification is robust because the groups are mutually exclusive and totally inclusive, so that each woman can be classified in a single group and each woman has a group where to be included $\mathrm{in}^{4}$. This allows overcoming a problem that frequently appears when CS is classified according to the indication. This classification is based on well-defined parameters that are easy to identify in each woman and her pregnancy instead of the CS indication, thus avoiding much confusion, discrepancy and variability, inherent to the CS indication. Four pillars form the basis of this classification: a) obstetric history; b) onset of labor; c) pregnancy category; and d) gestational age ${ }^{5}$.

\section{Description of the ten-group classification}

- Group 1: nulliparous women with a single cephalic pregnancy, 37 or more weeks of gestation, who have started labor spontaneously.

- Group 2: nulliparous women with a single cephalic pregnancy, 37 or more weeks of gestation, who either had labor induced or were delivered by cesarean section before labor.

- Group 3: multiparous women without previous cesarean section, with a single cephalic pregnancy, 37 or more weeks of gestation, who have started labor spontaneously.

- Group 4: multiparous women without previous cesarean section, with a single cephalic pregnancy, 37 or more weeks of gestation, who either had labor induced or were delivered by cesarean section before labor.

- Group 5: multiparous women with at least one previous cesarean section, with a single cephalic pregnancy, 37 or more weeks of gestation.

- Group 6: nulliparous women with a single breech pregnancy.

- Group 7: multiparous women with a single breech pregnancy, including those with previous cesarean sections.

- Group 8: all women with multiple pregnancies, including those with previous cesarean sections.

- Group 9: all women with a single pregnancy with a transverse or oblique lie, including those with previous cesarean sections.

- Group 10: all women with a single cephalic pregnancy with less than 37 weeks of gestation, including those with previous cesarean sections.

\section{Theoretical framework}

Cesarean section surgery emerged around 1880 to contribute to the reduction of maternal and fetal 
morbidity and mortality due to cephalopelvic disproportion, the mortality of which exceeded $80 \%$ in those days ${ }^{6}$. At the beginning of the $19^{\text {th }}$ century, it was implemented in cases of placenta previa or eclampsia, and it became an alternative procedure that was safer than craniotomy, symphysiotomy or than the use of high forceps in obstructed labor. Ever since, the indications did increasingly expand, with its use being increased. Early in the 1970s, the rate of CS in developed countries was around $5 \%$, and by the end of the 1990s, it had already exceeded $50 \%$ in some regions of the world. Many factors contributed to this increase: improvements in surgical and anesthetic techniques, reduction of postoperative complications in general, availability of antibiotics, nutritional and demographic factors, the perception of safety of the procedure by women and health personnel, implementation of a defensive medical practice against legal disputes, changes in health systems, request of the patient as a right to decide on the way to deliver her child, deferment of the age to get pregnant, increases in maternal body mass index, increase in the frequency of multiple pregnancies and decrease in vaginal deliveries after a first $\mathrm{CS}^{7}$.

\section{Classification systems for cesarean sections}

In general, classifications are based on the identification of different concepts that may have several parameters. Permutations of these parameters and their systematic organization generate specific groups or categories that share some properties, characteristics or defined qualities ${ }^{8}$. In order for data and information collected in health systems to be useful for improving people's health outcomes, it is necessary to organize them, and that is the role of classifications: organizing information and raw data to transform them into usable knowledge.

Each classification system has its objective, based upon which it is structured. The most frequent objective of classifications in medicine is to improve the quality of care and the results. Classification systems are successful only if the data or information that are collected and analyzed comply with the data collection principles ${ }^{9}$.

The information should be useful, carefully defined and collected, timely and available. The categories generated within classifications should also meet certain requirements: they must be mutually exclusive and totally inclusive. The classification should be easy to understand and implement, reproducible, and it should be able to be prospectively carried out in order to generate interventions.

There are several CS classifications; some are based on the indication and urgency of the procedure and on women's clinical and obstetric conditions and others are based on a variety of criteria ${ }^{10}$. In a review on the subject, 27 classifications were found, which were subjected to an evaluation process that took into account ease of application, clarity, reproducibility, the possibility of implementation, compliance with having mutually exclusive and totally inclusive categories, and the possibility of being prospectively applied. Only one classification obtained the highest possible score, the Robson 10-group classification, which belongs to the group of classifications based on women's characteristics $^{11,12}$. Among the classifications based on the urgency classification, the one that obtained the highest score (9 out of 14 possible points) was the van Dillen classification, and within the classifications based on the CS indication, two had the highest score $(9 / 14)$, Althabe's and Anderson's classifications.

\section{The Robson classification}

As the first reference of his classification, Dr. Michel Robson cites an article that he published in 200113; however, in a previous study, the author used the same categories of his classification system to describe the population and present the results of an audit process in 1996, although he does not give an identity to the classification model. Since its publication in 2001, the classification has been widely used in the world and has been applied both in institutional and regional fields and with different purposes $^{14}$.

The Robson classification is based on four obstetric concepts: obstetric history, onset of labor, pregnancy category and gestational age; with them, women are classified in one of 10 groups. Women are grouped, according to the category of pregnancy, as single pregnancy, with the presence of one fetus in the uterine cavity and multiple pregnancy when there is more than one; according to the fetal presentation at delivery, as cephalic or breech, oblique or transverse pregnancy; according to the obstetric history, as nulliparous if the patient does not have a previous delivery and multiparous if she has more than one previous delivery. In addition, history of abdominal surgery (with or without a uterine scar), onset of labor (medication-induced or spontaneous) and gestational age 
at delivery (full-term if gestation is $\geq 37$ weeks and preterm if $<37$ weeks) are considered.

Due to last menstrual period recognized lack of reliability and the margin of error of ultrasound to estimate it with the progress of gestation, if a newborn has an estimated gestational age of less than 37 weeks and a weight higher than the $95^{\text {th }}$ percentile for week $36(3093 \mathrm{~g})$, he/she will be reclassified in the newborn group of 37 weeks or more ${ }^{15}$.

The traditional classification of CS rates based on the indications for it is not recommended due to the difficulty for doing it prospectively without registration biases. The Robson classification is intended to identify groups with higher or lower probability of ending up in CS in order to take measures before the event occurs $^{16}$.

The characteristics of this classification system allow comparison within healthcare facilities over time, and between facilities, providing reasons to prospectively change practices in specific groups of women. It allows to identify which groups increase CS rates, recognizing in advance that some are susceptible to intervention and others not, and allows to re-evaluate the indication and pertinence of cesarean section, as well as to identify modifiable risk factors in these groups of patients, by means of which intervention strategies can be implemented.

Robson recognizes that his classification is an initial step and that, according to the results, additions must be implemented ${ }^{17}$. In the 2014 systematic review of the Robson classification, recommendations of users of this classification, who proposed group sub-classifications or new classifications and inclusion of other variables and maternal characteristics within each group, in addition to the need of health personnel training in order to obtain adequate data for patient classification, were considered ${ }^{18}$.

\section{Uses of the Robson classification}

The purpose the classification has most frequently been used for is to characterize the served population and to identify the groups that most contribute to the rate of CS or to its increase. This has been done for single institutions, for several institutions, for single cities, for single regions of a country, for multiple regions and even for several countries ${ }^{18}$. In some studies, in addition to this description, it is used to assess trends over time, both at institutional and population-based levels. Some authors go beyond the description and use the classification to make comparisons between institutions, to identify differences between them and to look for possible causes of variability in the rates of CS, and even to assess the effect of decreasing that variability both in single regions and between different regions or within population groups ${ }^{19}$. Some studies assess differences within groups and explore additional factors to those considered in the classification, such as explanations of the CS figures, their increases or their correlation with morbidity and mortality indicators ${ }^{20}$. It has also been used as a tool to standardize comparisons between institutions and to quantify the effect of interventions on decreasing the frequency of $\mathrm{CS}$. The classification has been used to meet objectives beyond CS, such as the study of severe obstetric morbidity, or to study specific subgroups of the classification. In Mexico, there is not a single published experience with the classification and, therefore, the rate of CS expected for an institution according to the type of served population using the classification has not been estimated and thus the rates have not been standardized.

The ten-group classification has been implemented in different obstetric units in the United Kingdom, Canada, Africa, Sweden, USA and Chile, among other countries.

In May 2015, WHO published a secondary analysis of two multinational surveys, the WHO Global Survey on Maternal and Perinatal Health (WHOGS, 2004-08) and the WHO Multicountry Survey on Maternal and Newborn Health (WHOMCS, 2010-11), where data from 21 included countries were analyzed to evaluate the CS operation trends. Data from these surveys were used to establish the average annual percentage change in CS rates by country, and the use of the Robson criteria was found to allow standardized data comparisons between countries and time points, and to identify the subpopulations that drive changes in CS rates, and thus their use is widely recommend. Having had a CS before is an increasingly important determinant of overall CS rates in countries with moderate or low human development index. Strategies to reduce the frequency of the procedure should include a reduction in the number of first cesarean sections and the best selection of cases for labor induction and CS in preterm pregnancies.

In Mexico, the use of a CS classification system has currently not been standardized, but some health institutions use the classification of emergency CS, depending on the degree of maternal or fetal compromise, with safety parameters being established according to the time between the diagnosis and the 
start of the surgical procedure. There are studies where the application of the Robson model for CS classification has been found to be easy and useful to identify women, procedures and possibly susceptible and priority intervention practices in order to have a rational CS rate adapted to the conditions of the institution and the women therein served.

\section{Problem statement}

when the pregnant woman and her relatives attend the emergency department of health facilities, they bring along a question about the birth of their child: is childbirth going to be by natural delivery or cesarean section?

By definition, childbirth is a physiological process that most times the pregnant woman experiences without problems, and if there are complications, they usually occur quickly and unexpectedly and should be foreseen by the professional. Since CS is a surgical medical intervention, it implies a risk that is inherent to the procedure; in this case, there can be complications derived from the surgical act or side effects of the anesthetic act, but when the intervention is indicated, these risks are lower than the benefits of performing the CS, which is not the case when the procedure is performed without a clear medical indication.

CS intervention, when justified, is efficacious for preventing maternal and perinatal morbidity and mortality. However, WHO reports that an ideal CS rate should not exceed $10-15 \%$, and that the benefits of CS are not demonstrated in patients in whom this procedure is unnecessary and entails immediate and subsequent risks, affecting the health of the mother-child dyad and future reproductive health of the woman.

The rate of CS in the world has clearly increased. The WHO indicates that Brazil is the country that is at the top, with $56 \%$ of deliveries with this procedure, followed by Egypt with $51.8 \%$, Turkey with $47.5 \%$, Italy with $38.1 \%$; several countries in Latin America show similar rates of CS.

In Mexico, there is an interest to study and expose this problem, which exists and alarmingly affects women's health. This almost usual CS use has increased to such an extent that tightening the controls on this type of surgeries has been decided in order to reduce their number. In our country, there is limited research on clinically unnecessary CS indicated by health professionals and, therefore, the reason for the medical decision on the preference of the pregnant woman is not known, including the decision on whether or not to avoid natural birth, the risk of which is sometimes exaggerated and that of cesarean section minimized, which affects the woman's reproductive health.

In addition, there is a void implied by not having a standardized system that internationally classifies CS rates, with trends and underlying causes, and hence their understanding, monitoring and comparison. In 2001, the Robson classification model, also known as the 10 Groups classification, was proposed, and after several studies that endorse it, it was incorporated by the WHO as a tool to control and evaluate CS rates, owing to its simplicity and the validity of its purpose, as well as to its ease of application and interpretation. Thus, the information collected in a standardized, uniform and reproducible manner is essential to the establishment of an improvement in health, with the purpose to optimize the use of CS intervention, and to evaluate and improve the quality of care.

CS is the most common surgical procedure in the entire world, and although it is true that it saves lives, very often it is practiced without a sustainable indication, which is an event that puts the pregnant woman and her newborn at risk of suffering some fatal health outcome. This is why it is essential to collect information on the use of CS at different levels of care, especially at secondary care, and thus being able to assess, improve and optimize the quality of care in order to identify the groups that most contribute to CS overall rates, as well as to implement practices within these groups of specific and prospectively identifiable women, which ultimately will provide us with a useful and clinically relevant classification system to improve the results.

The purpose of the present work is to determine the incidence of cesarean section using the Robson cesarean section classification, during the years 2014 to 2016 at Dr. Fernando Quiroz Gutiérrez General Hospital of the Institute of Social Security and Services for State Workers.

\section{Method}

The study was carried out at Dr. Fernando Quiroz Gutiérrez General Hospital, a secondary care center. It is a moderate and high complexity institution, responsible for the care of low, moderate and high-risk pregnant women and neonates, which serves patients who are affiliated to the healthcare system for State 
workers and who live in the western area of Mexico City. The study had a retrospective, cross-sectional and descriptive design. For the analysis of results, central tendency and dispersion measures were used; the results are presented in tables and graphs.

All women who attended the gynecology and obstetrics department for obstetric resolution by vaginal or cesarean section delivery between January 2014 and January 2016 were included. All patients with a pregnancy of more than 27 weeks were included. Data were retrospectively extracted from the hospital clinical records in the studied period. The ten-group classification proposed by Robson was used. The necessary information of each pregnant woman was obtained for this classification: a) single/multiple pregnancy; b) parity; c) onset of labor; d) gestational age; e) previous CS or uterine scar; f) fetal presentation and situation; maternal age was also taken into account. Onset of labor was considered spontaneous when there was no medical intervention to trigger the onset of uterine contractions, induced, when there was a pharmacological intervention for this purpose, and no labor, when CS was scheduled before one of both these events occurred. History of uterine scar included previous cesarean section or myomectomy. Gestational age was defined by the earliest ultrasound or amenorrhea onset recorded at the time of delivery. The diagnosis for the indication of pregnancy termination or for the performance of CS was taken from the clinical record. The rate of CS was calculated as the proportion of patients who had CS deliveries with regard to total deliveries recorded in the period of interest.

Patients were classified according to the ten mutually exclusive clinical groups described by Robson. The relationship between the patients belonging to each Robson group with respect to total served population was calculated ([no. of delivered women belonging to each group/total no. of delivered women, both vaginally and by CS] $\times 100$ ), which represents the relative size of each group.

The specific proportion of CS performed within each group was also calculated ([no. of CS performed in women belonging to each group/no. of deliveries, both vaginal and cesarean, belonging to that same group] $\times$ 100).

To quantify the contribution of each group to the general percentage of deliveries, the number of CS in the group was divided by total deliveries assisted in the period ([no. of total CS performed/total no. of deliveries, both vaginal and by CS] $\times 100$ ).

\section{Ethical aspects}

The present study is retrospective, based on patient charts and medical records, and the confidentiality, anonymity and rights of the participating women were preserved, with the investigation being regarded as free of risk, since the research subject does not suffer any damage as an immediate or delayed consequence of the study, according to the regulations of the General Statute of Health in Matters of Research, Title 2, on Ethical Aspects of Research in Human Beings, Chapter I, article 17-I.

\section{Results}

In the period from January 2014 to January 2016, 706 deliveries were assisted, out of which $65.29 \%$ were by cesarean section.

Women's average age was $31 \pm 4$ years, and mean gestational age was 38.5 weeks.

Among the participant women, $47 \%$ were primiparous and $53 \%$ were multiparous. In $62.2 \%$ of cases, labor started spontaneously, in $19.9 \%$, labor was induced, and of these, $17.9 \%$ corresponded to poor conditions for induction. At delivery, $91.78 \%$ of fetuses had a cephalic presentation. A history of uterine scar was recorded in $42.49 \%$ of patients.

The distribution by Robson clinical groups and their respective CS rates are summarized in table 1.

The indication to interrupt pregnancy by the abdominal route accounted for $67 \%$ of maternal causes and $33 \%$ of fetal causes. The most common maternal cause was iterative CS, with $42 \%$, followed by cephalopelvic disproportion with $38 \%$ (Fig. 1). The most common fetal cause was obstructed labor (dystocia) in $62 \%$ (Fig. 2).

\section{Discussion}

In recent years, there has been an increase in the rates of CS in most countries, with approximately 18.5 million CS being practiced annually, out of which half are considered unnecessary. In Mexico, a rate of $19 \%$ was reported in 1990 , which increased to $45 \%$ in 2009.

The use of the Robson classification model allowed identifying which groups are contributing the most to the proportion of CS in the hospital, which surpassed the $10-15 \%$ percentage established by WHO. In our hospital, an incidence of $65.29 \%$ was determined, which is well above the standard established by the 
Table 1. Cesarean sections distribution according to the Robson classification

\begin{tabular}{lccc}
\hline Groups & Group size & Cesarean sections specific proportion & $\begin{array}{c}\text { Contribution of cesarean sections in the group to } \\
\text { total deliveries (\%) }\end{array}$ \\
\hline Group 1 & $130(18.41 \%)$ & $68 / 130(52.30 \%)$ & 9.6 \\
Group 2 & $128(18.13 \%)$ & $98 / 128(76.56 \%)$ & 13.88 \\
Group 3 & $145(20.53 \%)$ & $29 / 145(20 \%)$ & 4.1 \\
Group 4 & $47(6.65 \%)$ & $30 / 47(63.82 \%)$ & 4.2 \\
Group 5 & $165(23.37 \%)$ & $150 / 165(90.90 \%)$ & 21.24 \\
Group 6 & $9(1.27 \%)$ & $9 / 9(100 \%)$ & 1.2 \\
Group 7 & $20(2.83 \%)$ & $20 / 20(100 \%)$ & 2.83 \\
Group 8 & $4(0.56 \%$ & $4 / 4(100 \%)$ & 0.56 \\
Group 9 & $33(4.6 \%)$ & $33 / 33(100 \%)$ & 4.6 \\
Group 10 & $20(3.54 \%)$ & $20 / 25(80 \%)$ & 2.83 \\
Total & 706 & $461 / 706$ & 65.29 \\
\hline
\end{tabular}

international community and causes a cost that exceeds the amount expected for the care of a delivery. Furthermore, a CS rate higher than $10 \%$ has been shown not to be associated with a decrease in maternal and neonatal mortality rates. Cesarean sections can cause significant complications and disabilities, sometimes permanent, and even death, especially in places where the facilities or the skills of health personnel to safely perform surgeries and to treat surgical complications are lacking. Ideally, CS should only be performed when they are necessary for medical reasons.

The groups that contributed the highest number of CS in the institution were Groups 5 (21.24\%), $2(13.88 \%)$ and $1(9.6 \%)$. Main indications for CS were iterative CS (42\%) and cephalopelvic disproportion $(38 \%)$. Group 5, which is the one that contributes the most to overall CS rate in institution, could be modified if a protocol for vaginal delivery care after a previous CS was established. In contrast, women with breech pregnancies (groups 6 and 7) and twin pregnancies (group 8) generally have a higher probability of CS delivery, along with those with transverse pregnancies (group 9), who have a clear obstetric indication for CS. The number of women belonging to these groups is a minority within our institution, and their contribution to the overall figure of CS is lower than that of other groups with less inherent risk for requiring surgical delivery.

Based on our findings, making the present results known within the institution is proposed, and for future studies, reviewing the indications and protocols for

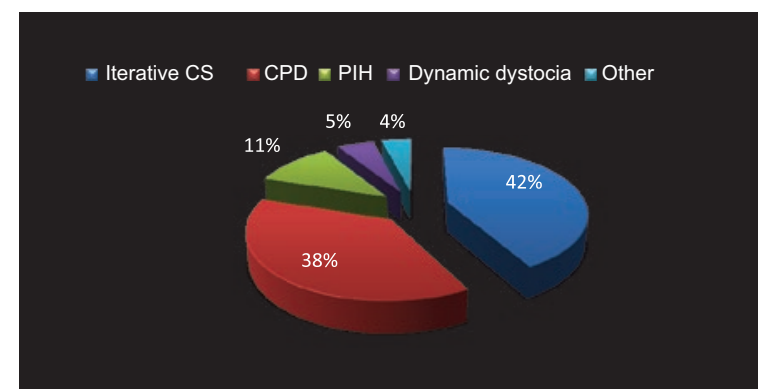

Figure 1. Diagnosed maternal causes for pregnancy interruption by CS (\%). CPD: cephalopelvic disproportion; PIH: pregnancy-induced hypertension.

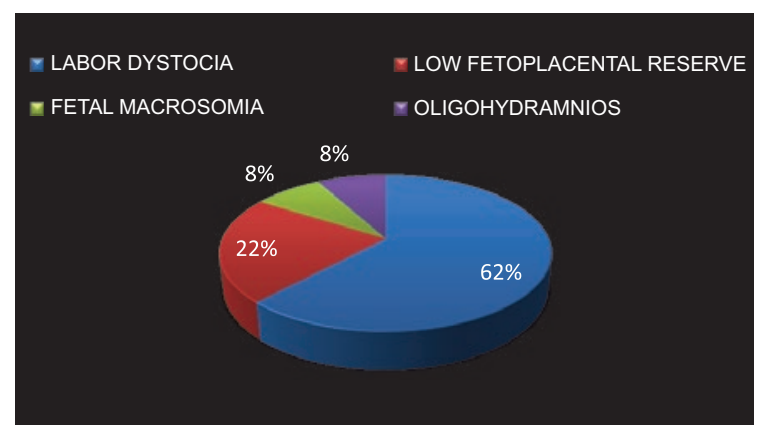

Figure 2. Fetal diagnoses that indicated pregnancy interruption by the abdominal route (\%).

induction of labor, the use of partogram and monitoring of fetal well-being, the indications to perform elective antepartum CS and the definition of failed induction of labor is suggested. In this sense, adoption and implementation of cervical ripening protocols, vaginal delivery care after CS and, with a lower expected impact, delivery care protocols for breech pregnancies is suggested. Finally, it is possible for strategies to reduce 
the CS rate to be implemented, such as a second expert opinion prior to performing the procedure. Correct information concerning gestational age and the indication for induction of labor, decreasing the number of previous CS in order to reduce the number of women with a previous history thereof, a description of the obstetric or perinatal indication of CS in preterm deliveries, and uniform reporting of fetal well-being by ultrasound and tococardiographic record, will allow adapting the strategies and interventions in order to rationalize the use of CS as a modality for delivery.

\section{Conclusions}

The Robson classification allows any health institution to identify the groups of women that most contribute to the rate of CS. It helps to establish groups of women with common obstetric characteristics undergoing CS that require intervention within an institutional protocol, and provides guidelines to define behaviors that impact on the overall rate of CS in an institution.

The groups of women with the highest specific CS rates are those with a previous CS (or previous uterine scar), nulliparous women, especially those undergoing induction of labor, and women with preterm pregnancies. These groups are susceptible to intervention in order to reduce the rate of CS within each one of them and impact on the institution's overall CS rate.

Making the results known to obstetricians-gynecologists of the institution is recommended, as well as implementing strategies that allow a reduction in CS overall rate, which should improve obstetric and perinatal care safety.

The WHO proposes using the Robson classification system as a global standard for assessing, monitoring and comparing CS rates within healthcare facilities over time, and between facilities. To assist healthcare facilities in adopting the Robson classification, WHO will develop guidelines for its use, implementation and interpretation, which will include a standardization of terms and definitions.

\section{References}

1. Palean L, Gibbons L, Chacón S, Ramil V, Belizan J. Tasa de cesáreas en dos hospitales privados con normativas diferentes: abierto y cerrado. Ginecol Obstet Mex. 2012;80:263-9.

2. Ruiz SJ, Espino SS, Vallejos PA, Duran AL. Cesárea: tendencias y resultados. Perinatol Reprod Hum. 2014;28:33-40.

3. Vélez PE, Tovar GVJ, Méndez VF, López LCR, Bustos ER. Incidencia, indicaciones y complicaciones de la operación cesárea en el Hospital de Ginecopediatría del IMSS de Hermosillo, Sonora. Bol Clin Hosp Infant Edo Son. 2012;29:58-64.

4. Schnapp CS, Sepúlveda E, Robert SJA. Operación cesárea. Rev Med Clin Condes. 2014;25:987-92.

5. Cabeza VPJ, Calvo PA, Betrán AP, Mas MMM, Febles BMM, Alcocer PX, et al. Clasificación de cesáreas por grupos de Robson en dos periodos comparativos en el Hospital de Manacor. Prog Obstet Ginecol. 2010;53:385-90.

6. Kazmi T, Saiseema S, Khan S. Analysis of cesarean section rate-according to Robson's 10-group classification. Oman Med J. 2012;27: 425-7.

7. Barcaite E, Kemekliene G, Railatete R, Bartuservicius A, Maleckliene L, Naisanskiene R. Cesarean section rates in Lithuania using Robson ten group classification system. Medicine. 2015;51:280-5.

8. Farine D, Shepherd D, Robson M. Classification of cesarean sections in Canada: the modified Robson criteria. J Obstet Gynaecol Can. 2012; 34:1133.

9. Ferreira EC, Costa ML, Cecattli JG, Haddad SM, Parpinelli MA, Robson MS. Robson ten group classification system applied to woman with severe maternal morbidity. Birth. 2015;42:1.

10. Betrán AP, Vindevoghel N, Souza JP, Guimezoglua AM, Torioni AM, Bhattacharya S. A systematic review of the Robson classification for cesarean section: what works, doesn't work and how to improve it. PLoS One. 2014;9:e97769.

11. Litorp H, Kidanto HL, Nystrom L, Darje E, Essen B. Increasing caesarean section rates among low-risk groups: a panel study classifying deliveries according to Robson at a University hospital in Tanzania. BMC Pregnancy. 2013;13:1-10.

12. Vogel JP, Betrán AP, Vindevoghel N, Souza JP, Torloni MR, Zhang J, et al. Use of the Robson classification to assess caesarean section trends in 21 countries: a secondary analysis of two WHO multicounty surveys. Lancet Global Health. 2015;3:260-7.

13. Betrán AP, Torloni MR, Zhang JJ, Guimezoglu AM, for the WHO working group on caesarean section. WHO statement on caesarean section rates. BJOG. 2016:123:667-70.

14. Muñoz-Enciso JM, Rosales Anjang E, Domínguez-Ponce G, Serrano-Díaz CL. Operación cesárea: ¿indicación justificante o preocupación justificada? Ginecol Obstet Mex. 2011:79:67-70.

15. Cyr RM. Myth of the ideal cesarean section rate: commentary and historic perspective. Am J Obstet Gynecol. 2006;194:932-6.

16. Zhang J, Troendle J, Reddy UM, Laughon SK, Branch DW, Burkman R, et al. Contemporary cesarean delivery practice in United States. Am J Obstet Gynecol. 2010; 203:326.e1-10.

17. Betrán AP, Vindenvoghel Nadia, Souza JP Gulmezoglu M, Torloni MR, et al. A systematic review of the Robson classifications for cesarean section. PLoS One. 2014;9:e97769.

18. Van Dillen J, Diesch M, Schutte J, Zwart J, Wolterbeek R, van Roosmalen J. Comparing grades of urgency for classification of cesarean delivery. Int J Gynaecol Obstet. 2009;107:16-8.

19. Althabe F, Belizán JM, Villar J, Alexander S, Bergel E, Ramos S, et al. Mandatory second opinion to reduce rates of unnecessary caesarean sections in Latin America: a cluster randomised controlled trial. Lancet. 2004;363:1934-40.

20. Anderson GM, Lomas J. Determinants of the increasing cesarean birth rate. Ontario data 1979 to 1982. N Engl J Med. 1984;311:887-92. 